\title{
Determination of Power-Quality Disturbances Using Teager Energy Operator and Kalman Filter Algorithms
}

\author{
Soo-Hwan Cho' ${ }^{1, *}$, Jeong-Uk Kim ${ }^{1}$, Il-Yop Chung ${ }^{2}$ and Jong-Hoon Han $^{3}$ \\ ${ }^{1}$ Department of Energy Grid, Sangmyung University, Seoul, 110-743, Korea \\ ${ }^{2}$ School of Electrical Engineering, Kookmin University, Seoul, 136-702, Korea \\ ${ }^{3}$ School of Electrical Engineering, Korea University, Seoul, 136-701, Korea
}

\begin{abstract}
With the development of industry, more large-scale non-linear loads are added to existing power systems and they cause the serious power quality (PQ) problems to the nearby sensitive installations more and more. To protect the important loads and mitigate the impact of PQ disturbances on them, various compensating devices are installed. One of the most important control skills used in the compensating equipment at the load side is how fast they can recognize or detect the discontinuous abnormal PQ events from the normal voltage signal. This paper deals with two estimation methods for the fast detection and tracking of general PQ disturbances: Teager Energy Operator (TEO), which is a non-linear operator and used for a short time energy calculation, and Kalman Filter (KF), which is one of the most universally used estimation techniques. And it is also shown how to apply the TEO and the KF to detect the PQ disturbances such as voltage sag, swell, interruption, harmonics and voltage fluctuation.
\end{abstract}

Keywords : Amplitude modulation, Detection, Kalman filter, Power quality (PQ), Teager-energy-operator (TEO).

\section{Introduction}

In the highly-modernized industrial era, there are increasing concerns about an unpolluted and clean quality of power, because many oversusceptible appliances based on the microprocessor can be easily damaged by the polluted voltage or current. The customers are willing to place some measures to protect their expensive electronics. For the fast compensation of PQ disturbances, it is very essential to detect the occurrence of disturbances as quickly as possible.

For the purpose, many advanced techniques widely used in the signal processing and automatic control fields such as Fast Fourier transform (FFT), Short-time Fourier transform (STFT), Wavelet transform (WT), Hilbert transform (HT), GaborWigner transform (GWT) and Kalman filter (KF) and so on, can be applied to the detection and classification areas of power quality disturbances.

Reference [1] provides a good and general review on various methodologies used for power quality analysis and event classification. However, since most of them are implemented in the computer based processor, they experience the relatively slow response by the calculation burdens.

This work presents the PQ disturbance detections by two different methods: Teager Energy Operator (TEO), which is a non-linear operator and used for a short time energy calculation,

Manuscript received Feb. 21, 2012; revised Mar. 1, 2012; accepted Mar. 5, 2012

*Corresponding author: Soo-Hwan Cho(shcho@smu.ac.kr)

(c) The Korean Institute of Intelligent Systems. All rights reserved. It is the extended version of a paper awarded as a best presentation in ISIS2011. and Kalman Filter (KF), which is one of the most universally used estimation techniques. Both of them have a common advantage of high speed detection ability.

At the next section, the algorithms of the TEO and the KF and their applications will be presented. In section III, we will explain the power quality events including voltage sag, swell, interruption, harmonics and voltage fluctuation to enhance the reader's understanding and simulate the case studies of PQ detections.

Finally, we summarize the simulation results and conclude the paper.

\section{TEO and Kalman Filter for PQ Event Detection}

\subsection{Teager Energy operator (TEO) Implementation}

TEO is a non-linear operator initially used for speech production modeling [2]. It can provide the fastest time response enabling to be implemented in the real-time and online measurement PQ event detection [3]. Some of previous papers have dealt with the TEO to apply to power quality detection and tracking [4]-[8]. References [4]-[6] focus on only tracking of voltage fluctuation, since a voltage fluctuation signal can be considered as one of the amplitude modulated signals. Reference [7] takes three different kinds of instantaneous frequency estimations such as TEO, Prony's method (PRONY) and modified covariance method (MCOV) into account and compares the performances of detecting instantaneous frequency in case of sag, swell and harmonics. It uses a discrete time energy separation algorithm I (DESA I) for AM-FM signal to analyze frequency components. Lastly, [8] 
deals with a combined TEO and threshold algorithm used for PQ events detection such as impulsive and oscillatory transients, notching and voltage sag, where a simple TEO calculation is only used to detect an instance when a event occurs.

In the previous papers, the TEO was mainly applied to studies on voltage fluctuation, because its characteristics are similar to an amplitude modulated signal.

The TEO of an oscillating signal, $x(t)=A \cos \left(\omega_{c} t+\theta\right)$, is defined as

$$
\Psi[x(t)]=[\dot{x}(t)]^{2}-x(t) \ddot{x}(t)=A^{2} \omega_{c}^{2}
$$

which is derived as follows:

$$
\begin{aligned}
& x(t)=A \cos \left(\omega_{c} t+\theta\right) \\
& \dot{x}(t)=-A \omega_{c} \sin \left(\omega_{c} t+\theta\right) \\
& \ddot{x}(t)=-A \omega_{c}^{2} \cos \left(\omega_{c} t+\theta\right) \\
& \Psi[x(t)]=[\dot{x}(t)]^{2}-x(t) \ddot{x}(t)=A^{2} \omega_{c}^{2}
\end{aligned}
$$

Its equivalent discrete version is given by

$$
\Psi[x[n]]=[x[n]]^{2}-x[n-1] x[n+1]
$$

Equation (3) shows that the TEO can be obtained by only three consecutive measurement data.

To trace the magnitude and frequency of a signal, another calculation to separate them from the TEO is needed. This is called as discrete energy separation algorithm, shortly DESA. The DESA has different forms, according to the characteristics of the analyzed signal. One is for the signal with a constant magnitude and a constant frequency. This case is suitable for representing PQ events with variations in the amplitude of a fundamental voltage signal such as voltage sag, voltage swell and interruption. Second is for the signal with variable amplitudes and a constant frequency. The other is for the signal with variable magnitudes and frequencies, which is a complicated case of a voltage fluctuation with frequency variation [5].

In this paper, the first two cases are considered because the events dealt in this work are sag, swell, interruption, harmonics and voltage fluctuation. The harmonics can be considered as a special case of variable amplitude and constant frequency signals.

The magnitude and frequency components of them are separated from the TEO by the following DESA equation.

$$
A[n]=\sqrt{\frac{\Psi[x[n]]}{\sin ^{2}\left(\Omega_{c}\right)}}
$$

$$
\Omega_{c}=\cos ^{-1}\left(1-\frac{\Psi[x[n]-x[n-1]]}{2 \Psi[x[n]]}\right)
$$

where $\mathrm{A}[\mathrm{n}]$ means the maximum value of an oscillating signal. In order to estimate the PQ disturbances, it needs to be converted to the RMS unit, that is, divided by $\sqrt{2}$.

Since the frequency component of (5) is expressed as a digital frequency, it needs to be converted into the continuous frequency component by dividing with $2 \pi / T$ ( $T$ : sampling interval(sec)).

\subsection{Kalman Filter Implementation}

Kalman filter (KF) is one of the most famous estimation skills. It is a very efficient control method used in the various application fields as well as power system [9-11].

$\mathrm{KF}$ is a repetitive calculation of the optimal estimation using a recursive least square method and the time-update prediction.

The state equation and the output equation of system are shown as below:

$$
\begin{gathered}
x_{k}=F_{k-1} x_{k-1}+w_{k-1} \\
y_{k}=H_{k} x_{k}+v_{k}
\end{gathered}
$$

The state equation provides time-information on the estimation of state variable $\left(\hat{x}_{k}\right)$ and the estimation error covariance $\left(P_{k}\right)$ and the output equation gives information to recursively calculate their updating values with a next-step measurement data $\left(y_{k}\right)$.

System variables are the state transition matrix $\left(F_{x}\right)$, the measurement matrix $\left(H_{k}\right)$, the state-variable noise covariance $\left(Q_{k}\right)$ and the measurement noise covariance $\left(R_{k}\right)$. With these data, we can compute the estimation of state variable $\left(\hat{x}_{k}\right)$, the estimation error covariance $\left(P_{k}\right)$ and a Kalman gain $\left(K_{k}\right)$.

The summarized process of the Kalman filter algorithm is shown as below.

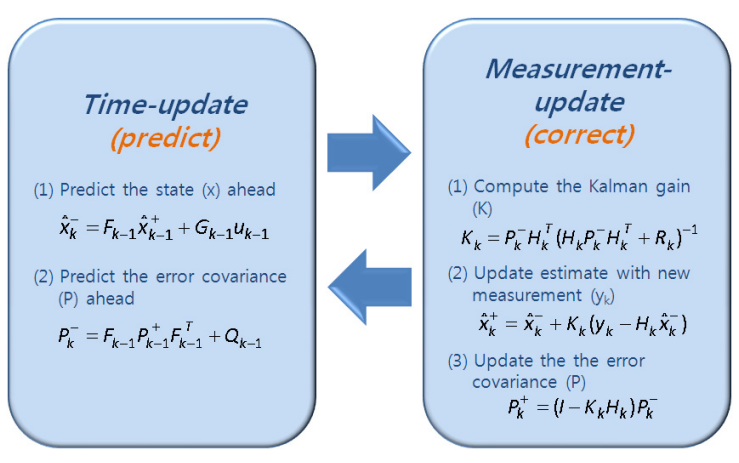

Fig. 1. Calculation Flow of Kalman Filtering

To apply the KF to the PQ detections, the state variables are given by $\left[x_{1}, x_{2}\right]^{T}=\left[V_{R}, 0\right]^{T}$ where $V_{R}$ is a voltage value in the RMS unit.

When the reference voltage signal $v(k T)$ is represented as 
$v(k T)=\sqrt{2} V_{R} \sin (2 \pi f k T)$, the state equation and measurement matrices are also given by

$$
\begin{gathered}
{\left[\begin{array}{l}
x_{1} \\
x_{2}
\end{array}\right]_{k}=\left[\begin{array}{ll}
1 & 0 \\
0 & 1
\end{array}\right]\left[\begin{array}{l}
x_{1} \\
x_{2}
\end{array}\right]_{k-1}+\left[\begin{array}{l}
\omega_{1} \\
\omega_{2}
\end{array}\right]_{k-1}} \\
H_{k}=[\sqrt{2} \sin (2 \pi f k T) \quad \sqrt{2} \cos (2 \pi f k T)]
\end{gathered}
$$

Measurement matrix $H_{k}$ should be synchronized with the measurement data $\left(y_{k}\right)$.

Kalman filter algorithm is written in MATALB as shown below [11]:

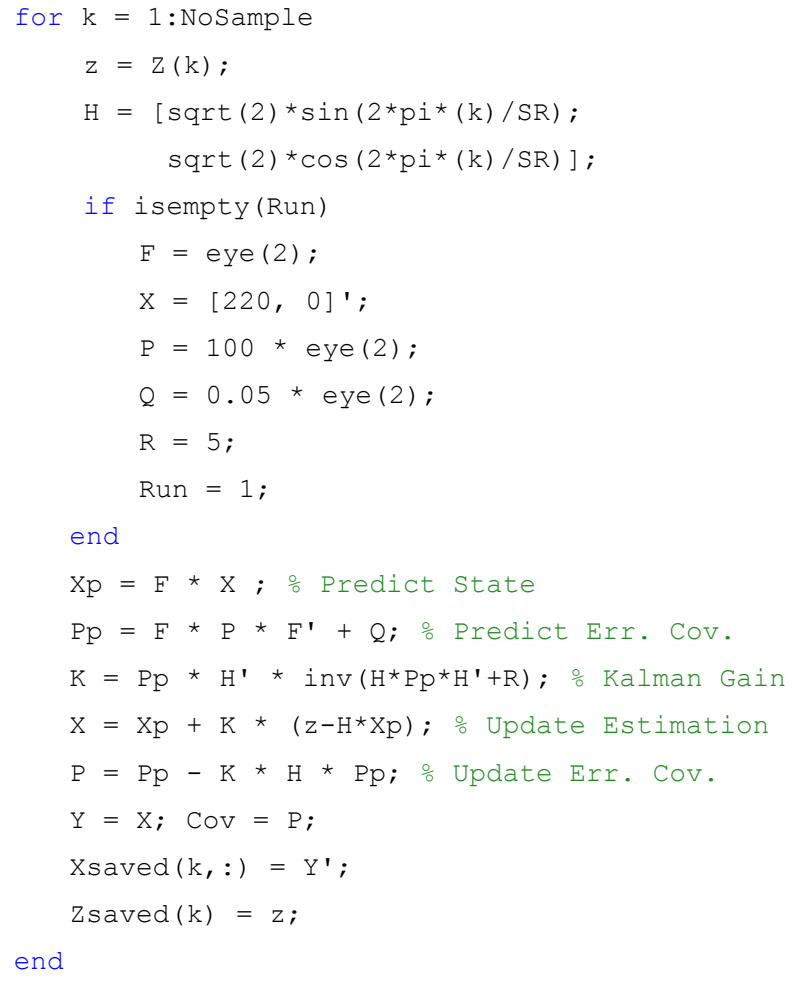

\section{Simulations}

\subsection{Voltage Sag and Swell}

According to the general understandings on power quality [12], voltage sag is one of the representative power quality problems and is defined as a short-duration (between 0.5 cycle and $1 \mathrm{~min}$ ) voltage reduction to between 0.1 and 0.9 p.u. in RMS magnitude. When it lasts longer than 1 minute, it is categorized into the under-voltage. Sags are caused by short circuits, overloads and starting transient of large-scale induction motors, which are frequently experienced in the normal power systems. This event can cause serious damage to several expensive and sensitive precision equipment including ASD(adjustable speed drive), manufacturing process and computer system. As a result, in order to minimize the damages, it is essential to detect the phenomenon as fast as possible, which can make some voltage compensating devices initialize the compensating actions.

Voltage swell is another issue of voltage variations and is defined as a short-duration (between 0.5 cycle and $1 \mathrm{~min}$ ) voltage increase over 1.1 p.u. in RMS magnitude. Swell is related to the system fault conditions, but is not as common as sag. It can also severely damage many industrial loads according to the system fault conditions and develop into the over-voltage.

A signal with a $20 \%$ sag and a $20 \%$ swell, each of which lasts for 6 cycles, is simulated by the magnitude separation from TEO and the KF as shown in Fig. 2. It shows that the detection using magnitude TEO is faster than when using Kalman filter by about $5 \mathrm{msec}$, which corresponds to 0.3 cycle.

\subsection{Interruption}

Interruption means complete loss of supplied voltage below 0.1 p.u. in RMS magnitude. According to the typical duration, interruptions are divided into 3 categories: instantaneous, momentary and sustained. The sustained interruption (longer than 1 minute) is mainly due to the outages of system components such as system faults, operations of protection relay and scheduled or emergency load shedding.

A signal with a $50 \%$ sag and a $5 \%$ interruption, each of which lasts for 6 cycles, is simulated by the magnitude separation from TEO and the KF as shown in Fig. 3. It also shows the faster detection performance of TEO than Kalman filter.
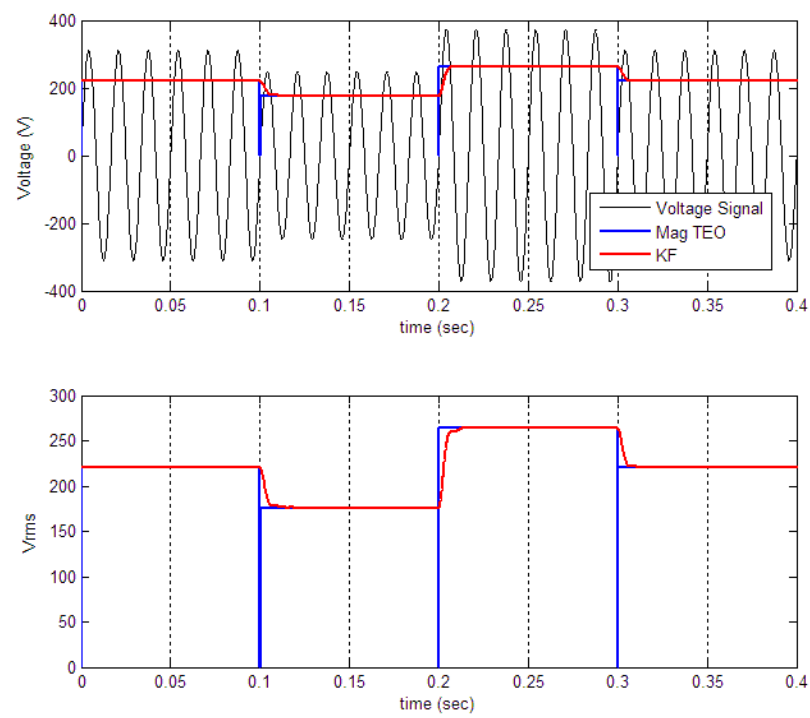

Fig. 2. Sag and swell detection by the magnitude separation from TEO (MagTEO) and Kalman filter (KF) 

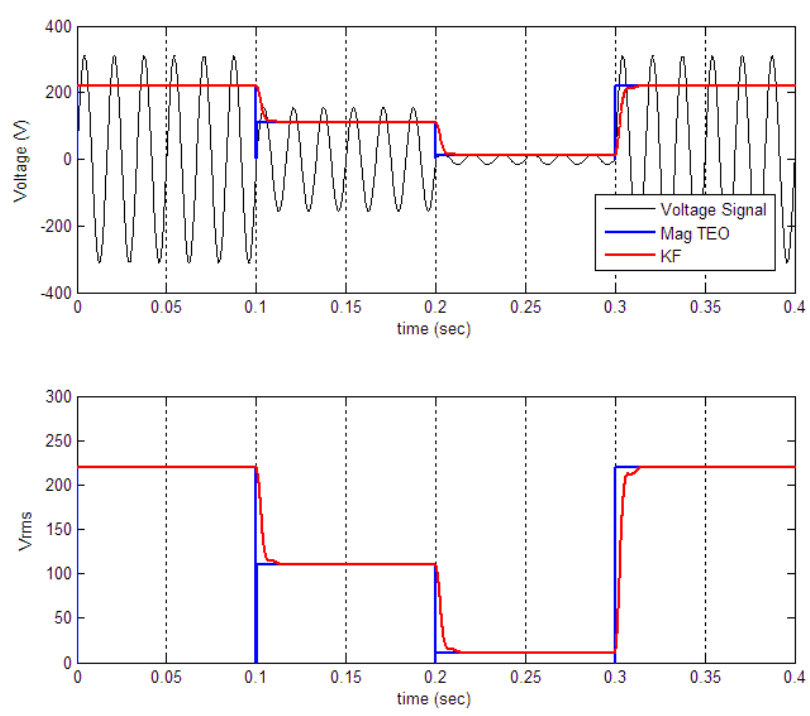

Fig. 3. Sag and interruption detection by the magnitude separation from TEO (MagTEO) and Kalman filter (KF)

\subsection{Harmonics}

Harmonics are very common events in the power supply systems. Because usual voltage and current signals are similar to the sinusoidal waveforms, they contain harmonic components whose frequencies are integer multiples of the fundamental one. Generally, the signals of voltage and current are easily polluted by the harmonic sources located throughout the power distribution systems, such as fluorescent lights, ASD for HVAC (Heat, Ventilation and Air-Conditioning) installations, 3-phase converters and arcing devices. Total harmonic distortion (THD) value is commonly used to evaluate the harmonic distortion levels.

Harmonic distortion has wide-spread influences in many power installations including motors, transformers, capacitors and telecommunications.

A signal containing a $5^{\text {th }}$ harmonic of $10 \%$ and a $7^{\text {th }}$ harmonic of $10 \%$ is simulated by the magnitude separation from TEO and the KF as shown in Fig. 4.

\subsection{Voltage Fluctuation}

Voltage fluctuation, also known as flicker, is a series of periodical variations of the voltage envelope. The term of flicker is used to expresses the effect of a magnitude modulated-voltage signal into the brightness of light loads, which is recognized by the visual sensation of end users. Consequently, the voltage fluctuation is a main cause of flicker and the flicker is a result of voltage fluctuation [13].

Main sources of voltage fluctuation are arc furnace, welding machine and successive switching operations. And it is closely related to inter-harmonics distortion.

A signal containing a $10 \mathrm{~Hz}$ and $20 \%$ sinusoidal fluctuation is simulated by the magnitude separation from TEO and the KF as shown in Fig. 5.
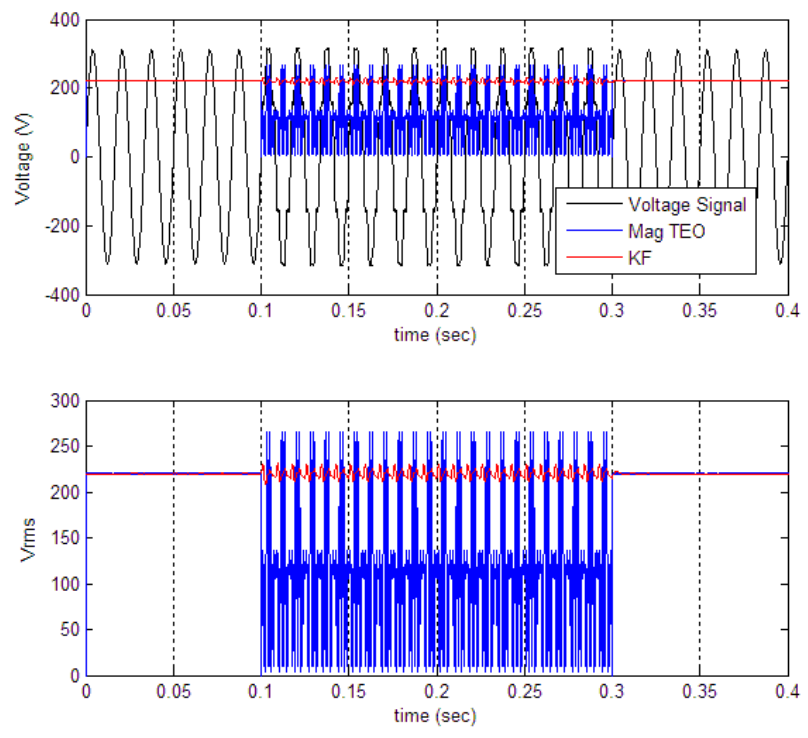

Fig. 4. Harmonics detection by the magnitude separation from TEO (MagTEO) and Kalman filter (KF)
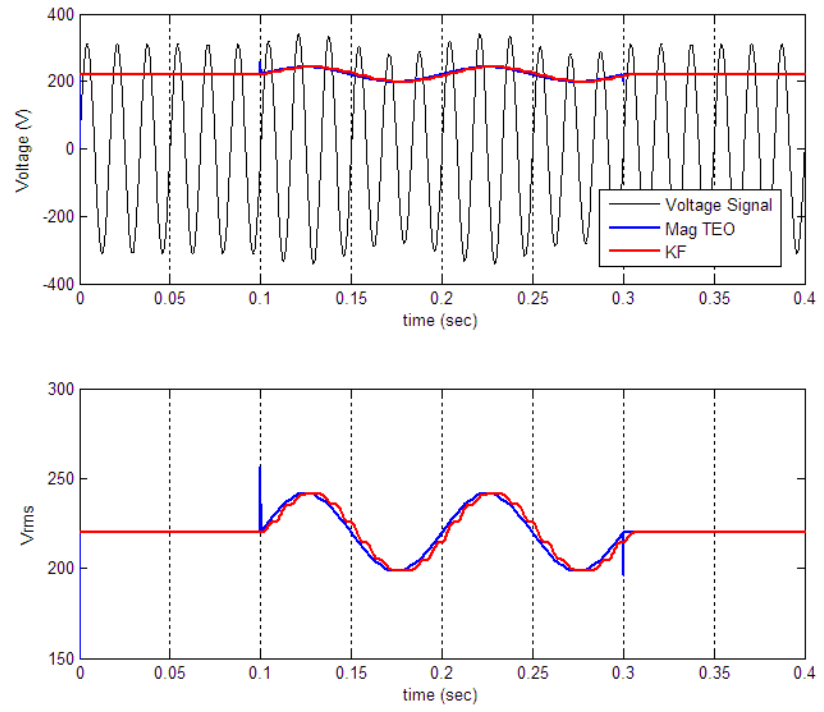

Fig. 5. Voltage fluctuation detection by the magnitude separation from TEO (MagTEO) and Kalman filter (KF)

\section{Conclusion}

In this work, the detection performances of various PQ disturbances by the Teager energy operator (TEO) and the Kalman filter (KF) are compared. A detection using the TEO is faster than the KF in many cases. The delay of Kalman filter is caused by the selection of covariance matrices of state variable noise and measurement noise.

As shown in the Fig. 2, 3 and 5, the notable advantage of TEO is that it can clearly indicate the starting and ending instances, which makes the recognition of event occurrences much faster. And the TEO gives a good performance of event 
detection for the voltage RMS variations such as sag, swell, interruption and voltage fluctuation.

However, the TEO has a disadvantage that it sensitively responses to the change of input values, as shown in Fig. 4.

A simple and fast calculation method by using the TEO and the DESA is very useful to detect some abnormalities in the power signal analysis. And the Kalman filter is also helpful, when it combined with the TEO and the DESA.

\section{References}

[1] D. Granados-Lieberman, R.J. Romero-Troncoso, R.A. Osornio-Rios, A. Garcia-Perez, E. Cabal-Yepez, "Techniques and Methodologies for Power Quality Analysis and Disturbances Classification in Power System: A Review," IET Generation, Transmission and Distribution, vol. 5, Iss. 4, pp. 519 - 529, 2011.

[2] H.M. Teager, "Some Observations on oral Air Flow During Phonation," IEEE Transactions on Acoustic, Speech and Signal Processing, vol. 28, Iss. 5, pp. 599 - 601, 1980.

[3] T.K. Abdel-Galil, I.O. Habiballah, "A Smart Power Quality Monitoring Strategy," AUSTRALASIAN UNIVERSITIES POWER ENGINEERING CONFERENCE (AUPEC 2005), Sep. 25-28, 2005, Hobart, Australia.

[4] M.A. Eldery, E.F. El-Saadany, M.M.A. Salama, "A Simple Energy Operator Computational Method for Voltage Flicker Assessment," IEEE Transactions on Power Delivery, vol. 21, no. 3, pp. 1743 -1750, 2006.

[5] T.K. Abdel-Galil, E.F. El-Saadany, M.M.A. Salama, "Energy Operator for On-line Tracking of Voltage Flicker Levels," IEEE POWER ENGINEERING SOCIETY WINTER MEETING, New York, USA, Jan. 27-31, 2002.

[6] T.K. Abdel-Galil, E.F. El-Saadany, M.M.A. Salama, "Online Tracking of Voltage Flicker Utilizing Energy Operator and Hilbert Transform," IEEE Transactions on Power Delivery, vol. 19, no.2, pp. 861 - 867, 2006.

[7] B.G. Iem, "Instantaneous Frequency Estimators as Voltage Disturbance Detectors," IEEE PES POWER SYSTEMS CONFERENCE AND EXPOSITION (PSCE 2006), Atlanta, USA, Oct. 29-Nov. 1, 2006.

[8] A. Bubasi, A.S. Yilmaz, K. Tufan, "Detection of Generated and Measured Transient Power Quality Events Using Teager Energy Operator," Energy Conversion and Management, vol. 52, Iss. 4, pp. 1959 - 1967, 2011.
[9] T.S. Jin and H. Hashimoto, "Simultaneous Localization and Mobile Robot Navigation Using a Sensor Network," International Journal of Fuzzy Logic and Intelligent Systems, Vol. 6, no. 2, pp. 161-166, 2006.

[10] D.S. Moon and S.H. Kim, "Design of Nonlinear Controller for Variable Speed Wind turbines based on Kalman Filter and Artificial Neural Network," Journal of Korean Institute of Intelligent Systems, vol. 20, no. 2, pp. 243-250, 2010.

[11] S.H. Cho, J.U. Kim and J.H. Han, "Pattern Estimation of PQ Disturbances using Kalman Filter," Proceeding of 2011 KIEE Summer Conference, Pyungchang, Korea, July 20-22, 2011.

[12] R.C. Dugan, M.F. McGranaghan, S. Santoso and H.W. Beaty, "Electrical Power Systems Quality," $2^{\text {nd }}$ Edition, McGraw-Hill Co., 2003.

[13] S.H. Cho, "Digital Implementation of the IEC flickermeter: Its Application for Measurement and Regulation Schemes in $220 \mathrm{~V} / 60 \mathrm{~Hz}$ Power System," Dissertation for the degree of Ph.D, 2009.

\section{Soo-Hwan Cho}

Professor of Sangmyung University

Research Area: Power Quality Analysis, Signal Processing, Measurement and Maintenance Method.

E-mail : shcho@smu.ac.kr

\section{Jeong-Uk Kim}

Professor of Sangmyung University

Research Area: Smart-grid Demand Response, Renewable Energy, Building and Traffic Control System.

E-mail : jukim@smu.ac.kr

\section{Il-Yop Chung}

Professor of Kookmin University

Research Area: Power Quality, Distributed Energy Resource, Renewable Energy.

E-mail : chung@kookmin.ac.kr

\section{Jong-Hoon Han}

$\mathrm{Ph} . \mathrm{D}$ candidate of Korea University

Research Area: Power System Analysis, Power Quality.

E-mail : hanpro_@korea.ac.kr 